\title{
Temporal bone imaging in osteogenesis imperfecta patients with hearing loss
}

F. Swinnen ${ }^{1}$, J. Casselman'2, P. Coucke ${ }^{3}$, C. Cremers ${ }^{4}$, E. De Leenheer ${ }^{1}$, I. Dhooge ${ }^{1}$

(1) Departement of Otorhinolaryngology, Ghent University (Hospital), Ghent, Belgium

(2) Department of Medical Imaging, AZ St-Jan Hospital, Bruges, Belgium

(3) Center for Medical Genetics, Ghent University (Hospital), Ghent, Belgium

(4) Department of Otorhinolaryngology, Radboud University Nijmegen Medical Center, Nijmegen, The Netherlands 


\section{Osteogenesis Imperfecta (OI)}

$\square$ 'Brittle bone disease'

a Connective tissue disease (1/20.000)

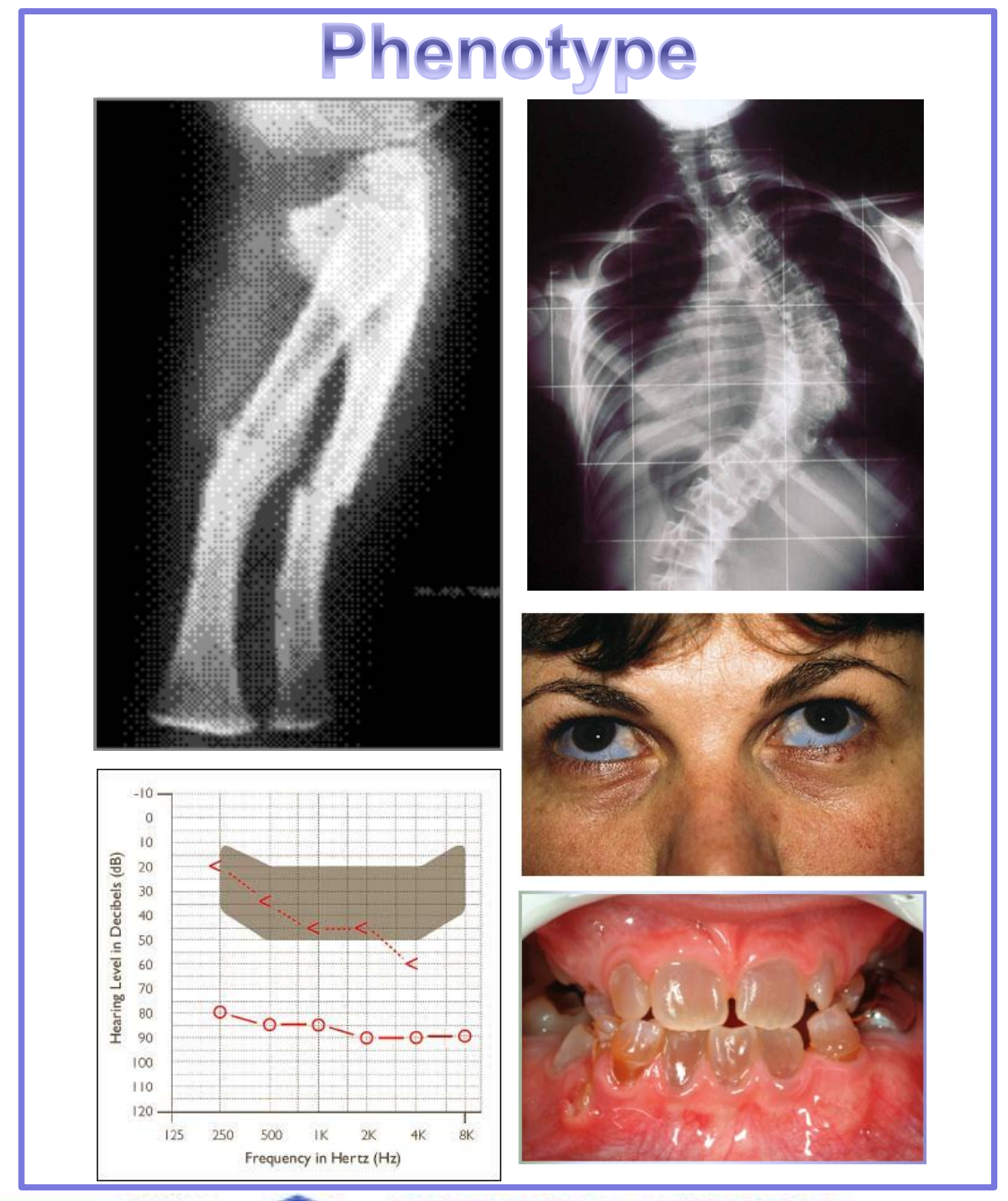

IIIIII

UNIVERSITEI

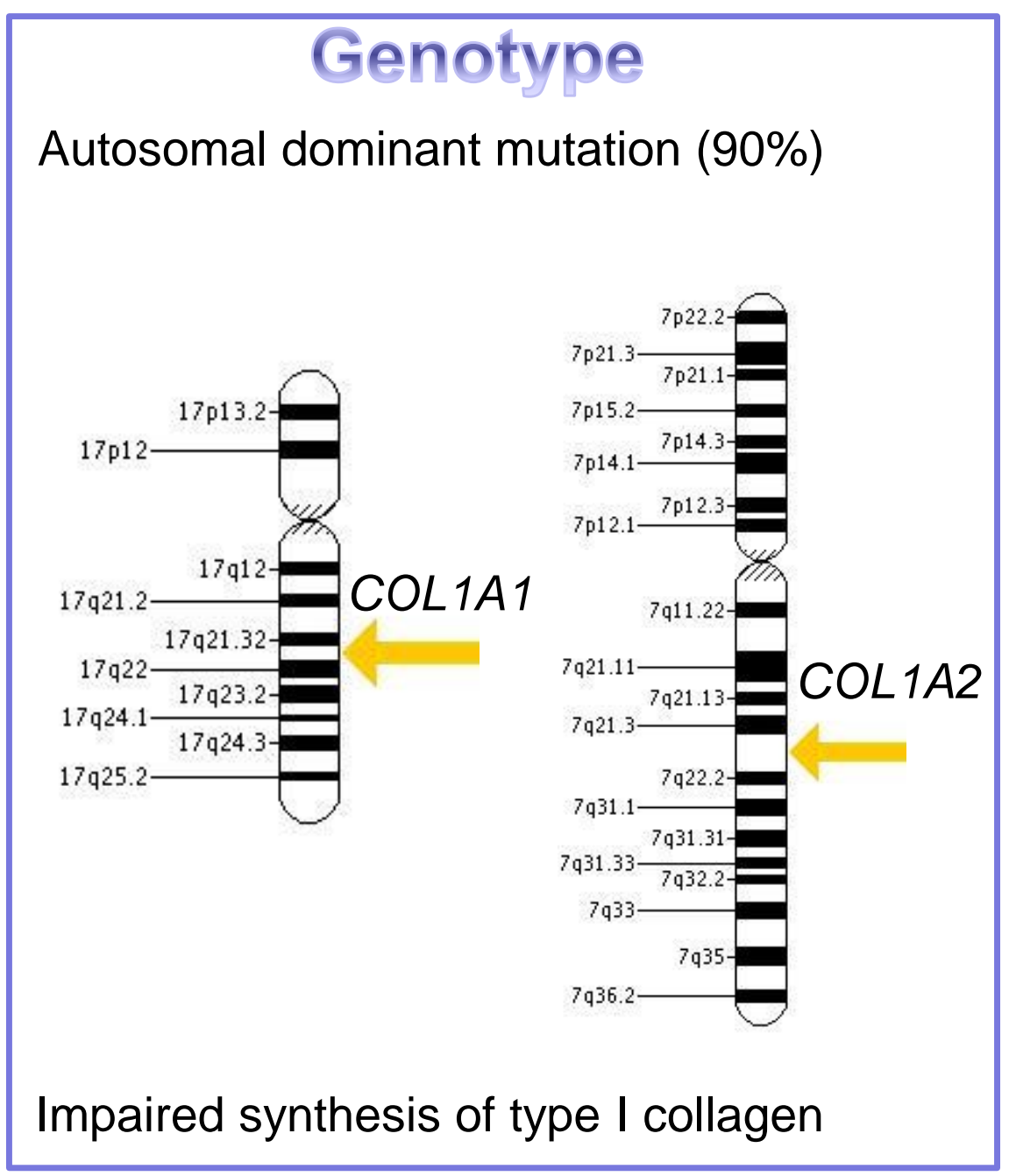

Universitair Ziekenhuis Gent 


\section{Ol - Hearing loss: prevalence and type}

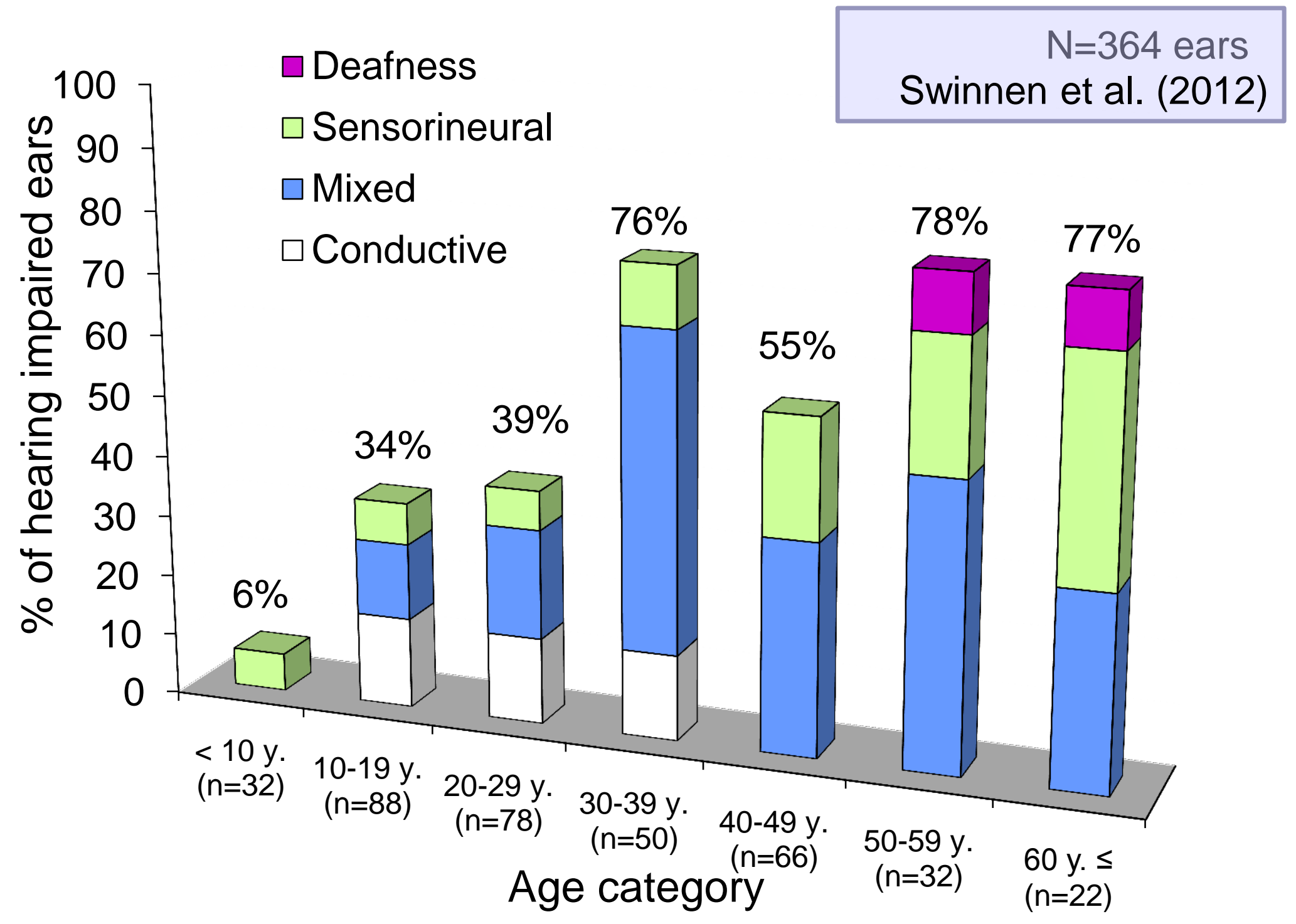




\section{Research aims}

Association between temporal bone imaging findings and audiometric results?
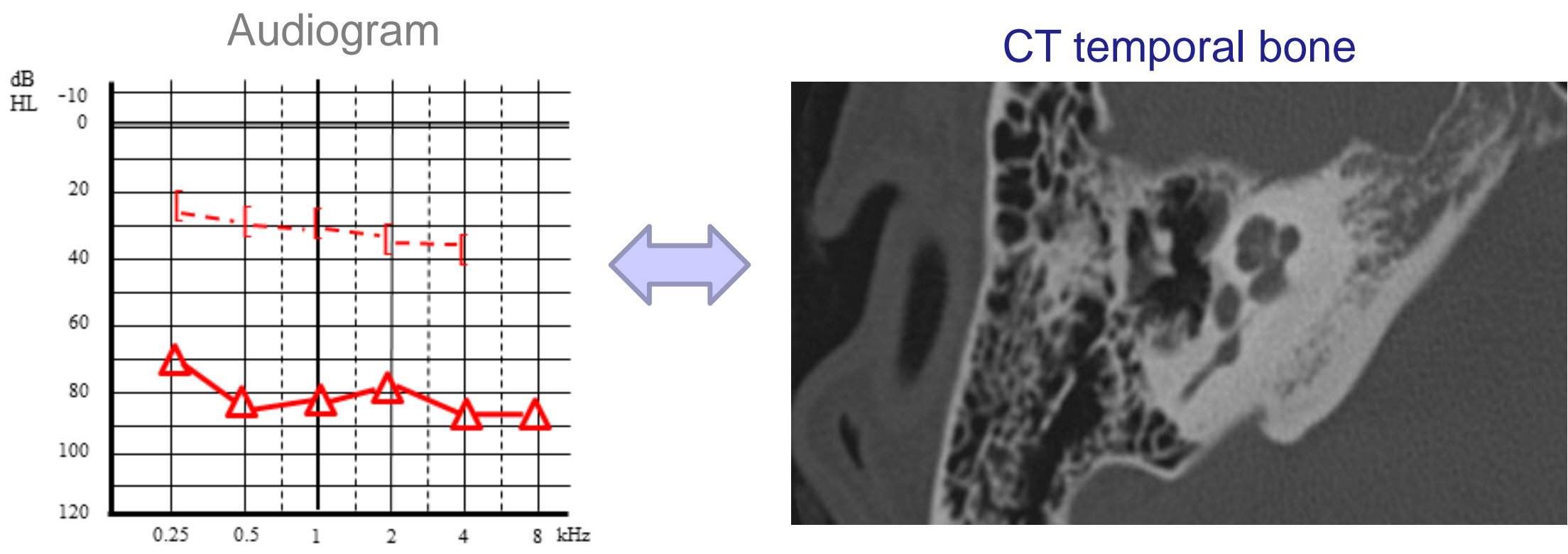


\section{Patients and materials}

$\square 17$ patients

- Age: 9-67 y.

- COL1A1 or COL1A2 mutation

$\square$ Retrospective study

- Audiograms

- CT images temporal bone (17 patients; 33 ears) 
Hearing loss in 31/33 ears

$\square$ Type of hearing loss

\begin{tabular}{|l|c|}
\hline & No. of ears \\
\hline Normal hearing & 2 \\
\hline Conductive hearing loss & 4 \\
\hline Mixed hearing loss & 20 \\
\hline Sensorineural hearing loss & 2 \\
\hline Deafness & 5 \\
\hline
\end{tabular}

$\square$ Hearing loss severity: mild to profound 


\section{Radiological evaluation: CT (1)}

Fenestral hypodensities in 26/33 ears (79\%)

\begin{tabular}{|l|l|l|}
\hline FAF & $25 / 33$ & $76 \%$ \\
\hline Oval window & $23 / 33$ & $70 \%$ \\
\hline Round window & $20 / 33$ & $61 \%$ \\
\hline
\end{tabular}
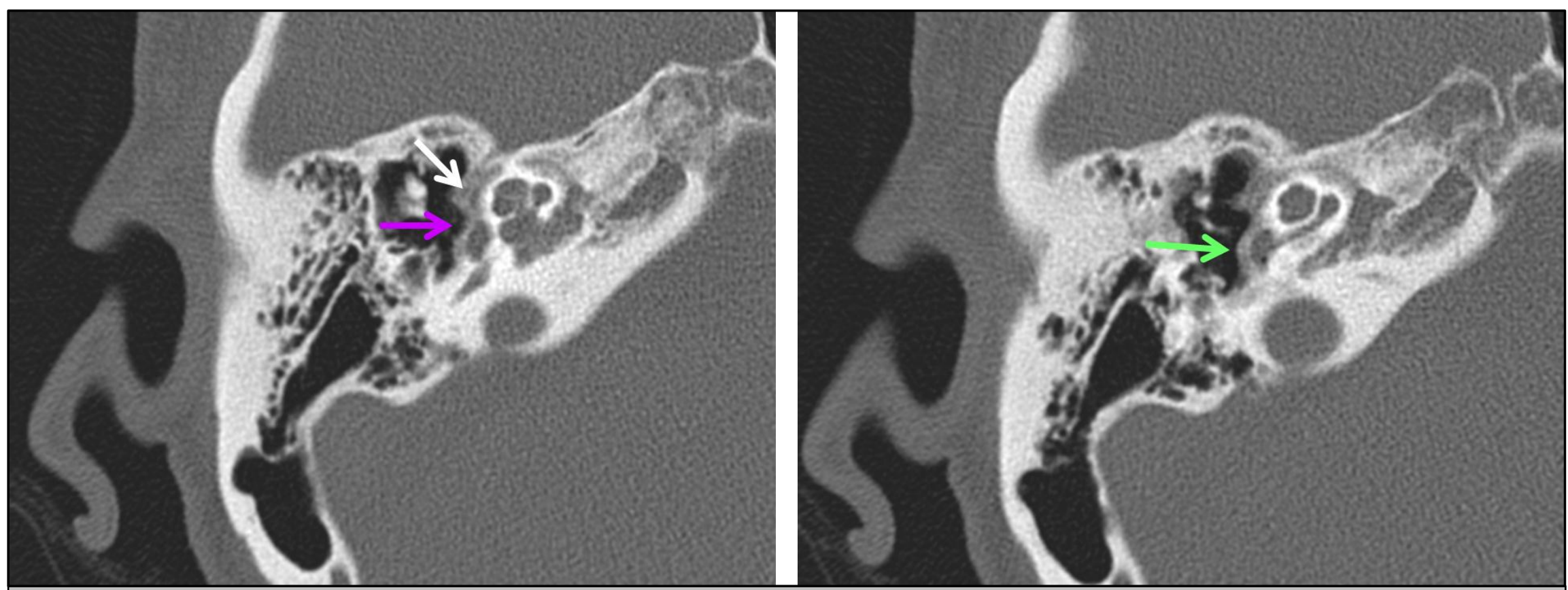

Axial CT images through right temporal bone (mixed hearing loss)
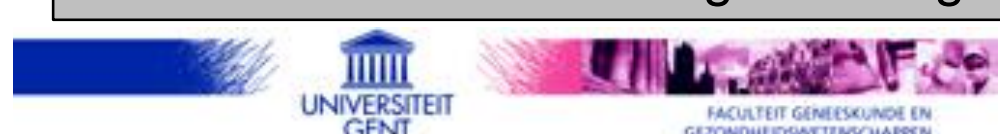


\section{Radiological evaluation CT (2)}

Retrofenestral hypodensities in 20/33 ears (61\%)

\begin{tabular}{|l|l|l|}
\hline Cochlear turns & $16 / 33$ & $49 \%$ \\
\hline $\begin{array}{l}\text { Hypodensity antero-inferior to internal } \\
\text { auditory canal }\end{array}$ & $16 / 33$ & $49 \%$ \\
\hline $\begin{array}{l}\text { Double ring sign } \\
\text { Facial nerve canal }\end{array}$ & $11 / 33$ & $33 \%$ \\
\hline Semicircular canals & $10 / 33$ & $30 \%$ \\
\hline
\end{tabular}


Radiological evaluation CT: retrofenestral hypodensities

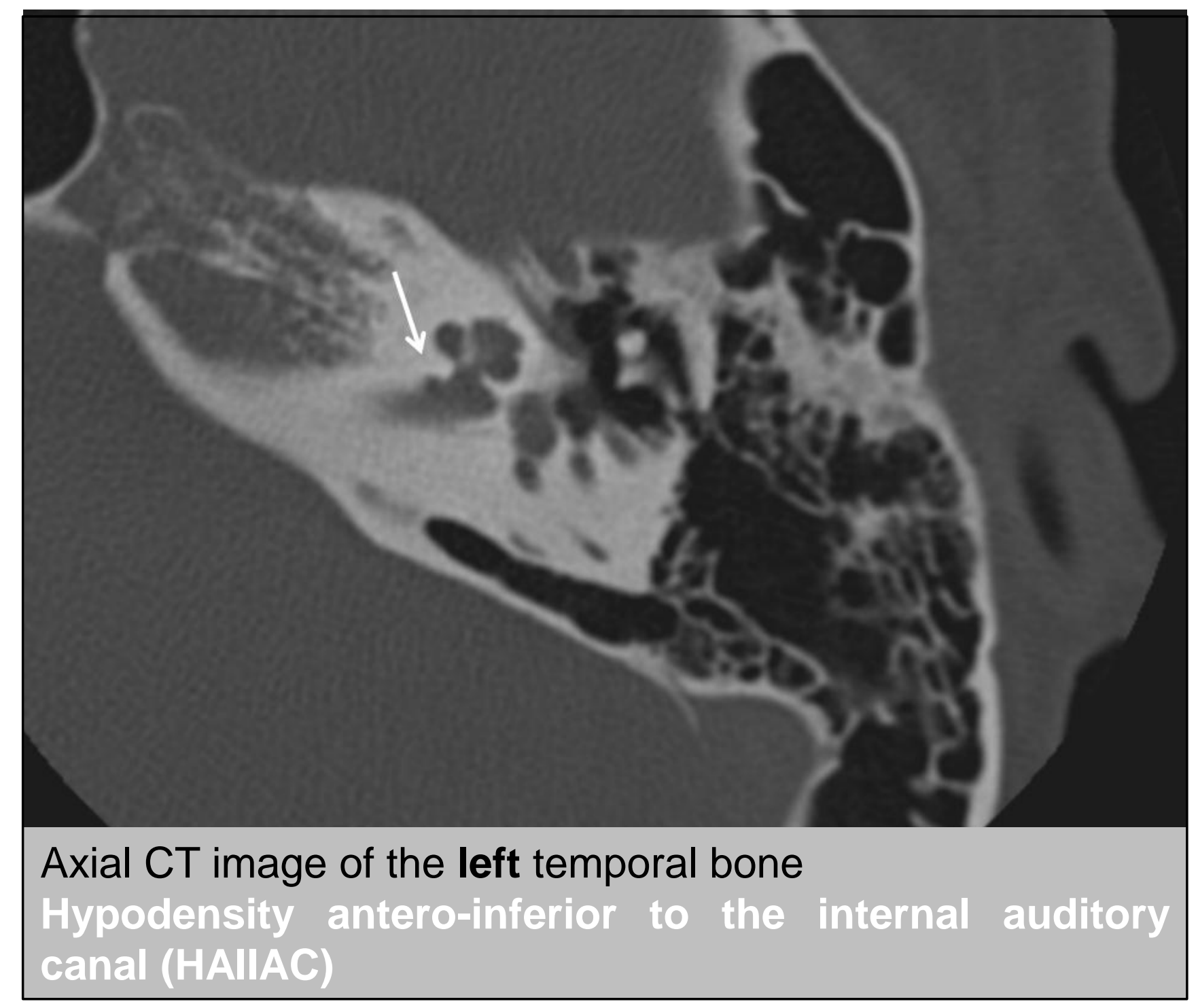


Radiological evaluation CT: retrofenestral hypodensities
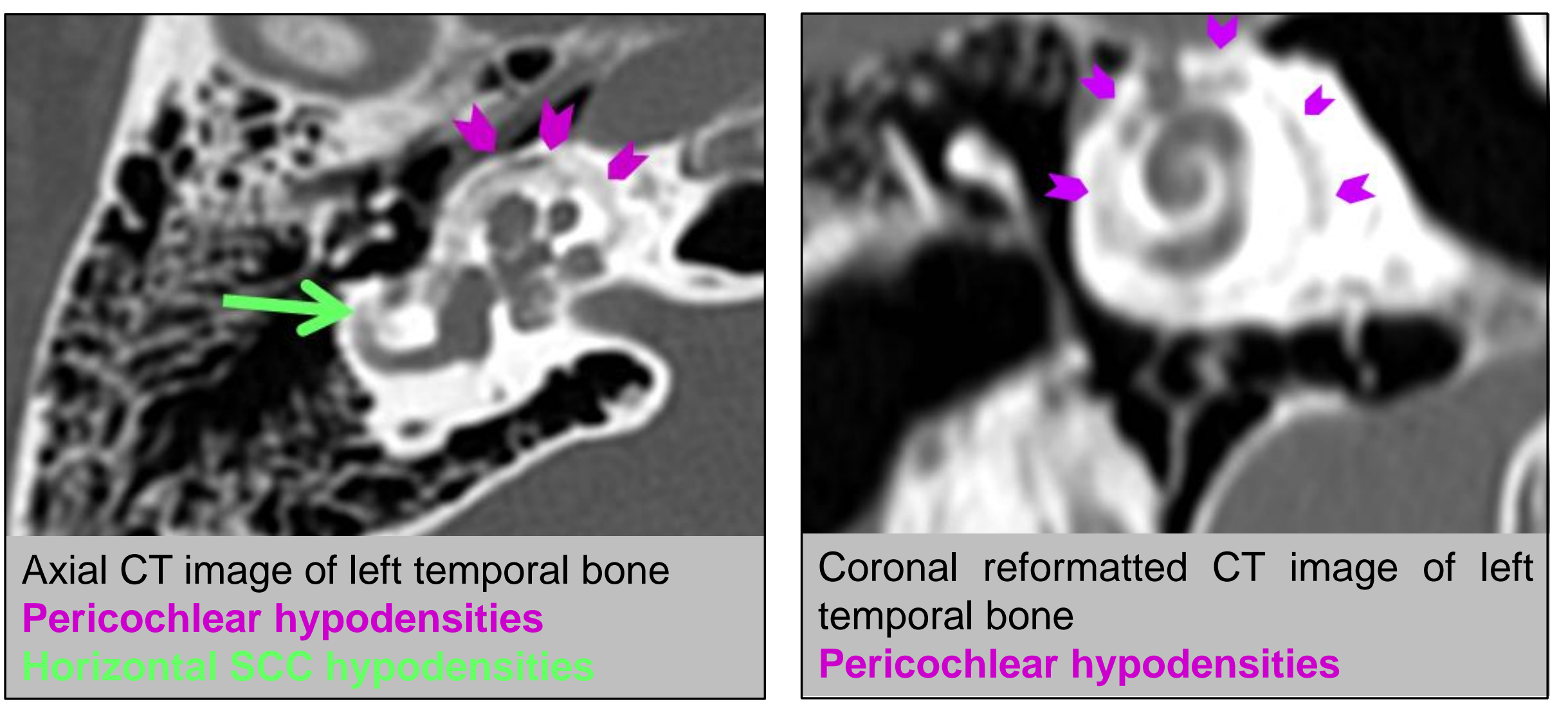


\section{Association audiometry - imaging findings}

\begin{tabular}{|c|c|c|c|c|}
\hline \multirow{2}{*}{$\begin{array}{l}\text { Audiometric } \\
\text { results }\end{array}$} & \multirow[t]{2}{*}{ No. of ears } & \multicolumn{3}{|c|}{ Hypodensities } \\
\hline & & None & Fenestral & $\begin{array}{c}\text { Fenestral + } \\
\text { retrofenestral }\end{array}$ \\
\hline Normal hearing & 2 ears (2 pts) & $1 / 2$ & $1 / 2$ & - \\
\hline Conductive HL & 4 ears ( 3 pts) & $\begin{array}{c}2 / 4 \\
(E V A+S S C C D)\end{array}$ & $2 / 4$ & - \\
\hline Mixed HL & 20 ears (12 pts) & $\begin{array}{c}2 / 20 \\
\text { (thick slices) }\end{array}$ & $3 / 20$ & $15 / 20$ \\
\hline SNHL & 2 ears (2 pts) & $1 / 2$ & - & $\begin{array}{c}1 / 2 \\
\text { (piston) }\end{array}$ \\
\hline Deafness & 5 ears ( 3 pts) & - & - & $5 / 5$ \\
\hline
\end{tabular}

The occurrence and location of the hypodensities affecting various structures of middle and inner ear corresponded to the expectations based on the type of hearing loss in $24 / 33$ ears $(73 \%)$. 


\section{Association hearing loss severity - affected structures}

Involvement of fenestral temporal bone structures in function of magnitude ABG

\begin{tabular}{|l|c|c|c|c|c|l|}
\hline \multirow{2}{*}{ Structure } & \multicolumn{7}{|c|}{ ABG } \\
\cline { 2 - 8 } & $<15 \mathrm{~dB}$ & $15-25 \mathrm{~dB}$ & $25-35 \mathrm{~dB}$ & $35-45 \mathrm{~dB}$ & $>45 \mathrm{~dB}$ & Total \\
\hline FAF & $1 / 4$ & $5 / 7$ & $4 / 7$ & $5 / 5$ & $4 / 4$ & $19 / 27^{*}$ \\
\hline Oval window & $2 / 4$ & $3 / 7$ & $5 / 7$ & $5 / 5$ & $3 / 4$ & $18 / 27^{*}$ \\
\hline Round window & $1 / 4$ & $3 / 7$ & $4 / 7$ & $4 / 5$ & $3 / 4$ & $15 / 27^{*}$
\end{tabular}

${ }^{*} 6$ ears with undeterminable ABG excluded.

$\square$ Significant positive correlation between the number of affected fenestral structures on CT and the magnitude of ABG on the audiogram $(r=0.464 ; p<0.05)$

$\square$ No association between magnitude of $A B G$ and involvement of a specific structure 


\section{Association hearing loss severity - affected structures}

$\square$ Involvement of retrofenestral temporal bone structures in function of the average bone conduction threshold

\begin{tabular}{|l|c|c|c|c|c|c|}
\hline \multirow{2}{*}{ Structure } & \multicolumn{6}{|c|}{ Average bone conduction threshold } \\
\cline { 2 - 8 } & $\begin{array}{l}<15 \\
\text { HL }\end{array}$ & $\begin{array}{l}15-25 \\
\text { dB HL }\end{array}$ & $\begin{array}{l}25-35 \mathrm{~dB} \\
\text { HL }\end{array}$ & $\begin{array}{l}35-45 \\
\text { dB HL }\end{array}$ & $\begin{array}{l}>45 \mathrm{~dB} \\
\text { HL }\end{array}$ & Total \\
\hline Cochlear turns & $0 / 6$ & $6 / 12$ & $2 / 4$ & $3 / 5$ & $5 / 6$ & $16 / 33$ \\
\hline Facial nerve canal & $0 / 6$ & $4 / 12$ & $1 / 4$ & $3 / 5$ & $2 / 6$ & $10 / 33$ \\
\hline Semicircular canals & $0 / 6$ & $1 / 12$ & $1 / 4$ & $0 / 5$ & $3 / 6$ & $5 / 33$ \\
\hline HAllAC & $0 / 6$ & $9 / 12$ & $3 / 4$ & $2 / 5$ & $2 / 6$ & $16 / 33$ \\
\hline Double ring sign & $0 / 6$ & $4 / 12$ & $0 / 4$ & $2 / 5$ & $5 / 6$ & $11 / 33$ \\
\hline
\end{tabular}

$\square$ Significant positive correlation between the number of affected retrofenestral structures on CT and the average bone conduction threshold determined by audiometry $(r=0.471 ; p<0.05)$

$\square$ No association between magnitude bone conduction threshold and involvement of a specific structure 
Similarities with otosclerosis (otospongiosis)

$\square$ Type of hearing loss in Ol

associated with location of hypodens areas:

- Conductive loss: fenestral

- Mixed hearing loss: fenestral + retrofenestral

$\square$ Severity of the conductive/sensorineural component associated with extent of hypodensities affecting temporal bone structures

$\square$ ? Severe OI (type III): higher risk on

- EVA

- dehiscent SCC 
$\square$ Surplus value of temporal bone imaging in OI:

- Confirmation of diagnosis of Ol

- Etiology of hearing loss

- Extent of temporal bone affection

- Unsatisfactory hearing gain after stapes surgery

- Preoperative evaluation in stapes surgery and cochlear implantation

$\square$ Submillimetric spatial resolution recommended 
THANK Pas 